\title{
Asociación diferencial entre el metabolismo regional de la corteza prefrontal y las estructuras límbicas y el tiempo de abstinencia en individuos dependientes de sustancias
}

\section{Differential association between the regional metabolism of the prefrontal cortex and limbic structures, and the time of abstinence in substance dependent individuals}

\author{
Laura Moreno-López*; María José Fernández-Serrano**; Emmanuel Andreas Stamatakis***; \\ Manuel Gómez-Río****; Antonio Rodríguez-Fernández****; Miguel Pérez-García*******,******; \\ ANTONIO Verdejo-GarCía*,*****, *******
}

\begin{abstract}
*Departamento de Personalidad, Evaluación y Tratamiento Psicológico. Universidad de Granada. España; **Departamento de Psicología. Universidad de Jaén. España; *** Divison of Anaesthesia. University of Cambridge, Cambridge. Reino Unido; ****Servicio de Medicina Nuclear del Hospital Virgen de las Nieves de Granada. España; *****Instituto de Neurociencias Federico Olóriz, Universidad de Granada. España; ******CIBERSAM. Universidad de Granada. España; *******School of Psychology and Psychiatry, Monash University, Melbourne, Australia.
\end{abstract}

\section{Resumen}

A pesar de la existencia de numerosos estudios de neuroimagen cerebral demostrado la presencia de alteraciones del metabolismo cerebral en consumidores de drogas, pocos estudios han tratado de evaluar la asociación entre la duración de la abstinencia y el metabolismo cerebral de los consumidores. El objetivo de este estudio fue investigar la asociación entre el metabolismo cerebral de la glucosa (medido con 18-F fluor-desoxi-glucosa y tomografía por emisión de positrones) y la duración de la abstinencia en un grupo de 49 policonsumidores de drogas con abstinencia prolongada. Las imágenes fueron preprocesadas y analizadas usando SPM5 y SPSS 15. Tras el pre-procesamiento de las imágenes se extrajo el nivel del consumo de glucosa en un conjunto pre-establecido de regiones de interés y se llevaron a cabo correlaciones bivariadas entre el metabolismo de estas regiones y la duración de la abstinencia de los participantes. Se encontró una correlación negativa entre la duración de la abstinencia y el metabolismo regional de la amígdala y el hipocampo bilateralmente y una correlación positiva entre la duración de la abstinencia y la porción opercular del giro frontal inferior izquierdo. Las asociaciones encontradas sugieren la implicación diferencial de estas estructuras en el mantenimiento de la abstinencia y subrayan la necesidad de trabajar la regulación del estrés, el craving y el control de la conducta incluso tras importantes periodos de abstinencia.

Palabras clave: adicción, PET, abstinencia, hipocampo, amígdala, giro frontal inferior.

\begin{abstract}
Despite the existence of numerous neuroimaging studies demonstrating significant brain functional alterations in substance users, only a few studies have tried to analyze the association between the duration of abstinence and brain metabolism within substance users. The aim of this study was to examine the association between resting-state regional brain metabolism (measured with 18F-fluordeoxyglucose Positron Emission Tomography (FDGPET) and duration of drug abstinence in a sample of 49 abstinent polysubstance users. PET images were pre-processed and analyzed using SPM5 and SPSS 15. After image pre-processing, the level of glucose uptake in a pre-established set of regions of interest was extracted and bivariate correlations between this and the duration of abstinence of the participants were conducted. Results showed a negative correlation between duration of abstinence and the amygdale and the hippocampus bilaterally and a positive correlation between duration of abstinence and the left inferior frontal operculum. The associations found suggest different involvement of these structures in maintaining abstinence and emphasize the need to work on stress regulation, craving and behaviour control even after significant periods of abstinence.
\end{abstract}

Key words: addiction, PET, abstinence, hippocampus, amygdale, inferior frontal gyrus. 
L a evidencia indica que los primeros contactos con las drogas producen efectos placenteros en las personas que las consumen y durante mucho tiempo, estos efectos han sido asociados con el incremento de los niveles de dopamina en el circuito mesolímbico dopaminérgico. Utilizando los mismos mecanismos fisiológicos que los reforzadores naturales, las drogas actúan sobre este sistema pero a distintos niveles. Así, la cocaína, las anfetaminas y la nicotina producirían la liberación de dopamina en el núcleo accumbens, los opiáceos producirían la activación de los receptores de péptidos opioides en el área tegmental ventral y el núcleo accumbens y el alcohol produciría la activación de sistemas GABA en el núcleo accumbens y la amígdala (Camí y Farré, 2003). Sin embargo, a medida que el consumo recreativo comienza a ser adictivo, se producen un conjunto de neuroadaptaciones en las regiones asociadas con los efectos reforzantes de las drogas y en aquellas conectas neuroanatomicamente con estas que conllevan el paso del consumo de drogas motivado por sus efectos reforzantes, al consumo impulsivo y finalmente compulsivo, el consumo crónico y la recaída (Goldstein y Volkow, 2011). El drogodependiente ya no consume la droga por sus efectos reforzantes sino para reducir el craving o el deseo intenso por consumirla, y lo que es más importante, lleva a cabo esta conducta a pesar de conocer las terribles consecuencias negativas que se derivan de la misma (Volkow y Lee, 2004).

La mayoría de los estudios que se han llevado a cabo en el campo de las adicciones se han centrado en la etiología del trastorno y en los cambios producidos a nivel cerebral como resultado del consumo de las diferentes drogas y sólo recientemente algunos investigadores han empezado a estudiar las áreas implicadas en el mantenimiento de la abstinencia o en la recuperación del paciente drogodependiente (Connolly, Foxe, Nierenberg, Shpaner y Garavan, 2012; Garavan y Weierstall, 2012).

El extraordinario desarrollo de las técnicas de neuroimagen cerebral ha permitido investigar con mayor precisión la naturaleza, localización y extensión de dichas neuroadaptaciones y en muchas ocasiones, se ha mostrado como la herramienta más eficaz para detectar las alteraciones producidas a nivel cerebral en consumidores de ciertas sustancias psicoactivas (p.e. Barrós-Loscertales et al., 2011; Tapert et al., 2007). De entre las diferentes técnicas utilizadas, el uso de la Tomografía por Emisión de Positrones (PET) en combinación con el trazador 18-Flúor-Desoxi-Glucosa (18FDG) ha demostrado ser una herramienta de gran utilidad en la identificación, localización y cuantificación del consumo de glucosa a nivel cerebral. Sin embargo, no existen hasta el momento estudios que hayan tratado de evaluar de forma sistemática la asociación entre el metabolismo cerebral y la duración de la abstinencia

Este estudio tuvo como objetivo principal investigar la asociación entre la duración de la abstinencia y el metabo- lismo cerebral de la glucosa en un grupo de policonsumidores de drogas con prolongada abstinencia. Basándonos en la implicación diferencial de las áreas del sistema mesolímbico dopaminérgico y las estructuras prefrontales en el proceso adictivo y en el mantenimiento de la abstinencia, esperamos encontrar una asociación diferencial entre la duración de la abstinencia y el metabolismo de la glucosa de estas áreas. En concreto, esperamos encontrar una asociación negativa entre la duración de la abstinencia y el metabolismo cerebral de las estructuras del sistema mesolímbico dopaminérgico y una asociación positiva entre la duración de la misma y el metabolismo cerebral de estructuras prefrontales.

\section{Método}

\section{Participantes}

Las características de los participantes de este estudio han sido descritas en dos estudios previos (ver Moreno-López et al., 2012a, 2012b). Brevemente, los participantes fueron reclutados durante su estancia en la comunidad terapéutica de "Proyecto Hombre" en Granada. Este centro proporciona un entorno controlado para el tratamiento de los trastornos por consumo de sustancias. Los criterios de selección fueron (i) cumplir los criterios de dependencia fijados por el DSM-IV al inicio del tratamiento - evaluados por la versión clínica de la entrevista clínica estructurada para los trastornos del eje I del DSM-IV (First et al., 1997), (ii) ausencia de trastornos del estado de ánimo o trastornos de personalidad comórbidos al comienzo del tratamiento, (iii) ausencia de lesiones cerebrales o trastornos neurológicos y (iv) presentar una duración mínima de abstinencia de 15 días antes de la realización de la prueba. La abstinencia de los participantes era comprobada a través de pruebas de orina realizadas semanalmente de forma aleatoria. Ninguno de los participantes mostró síntomas de abstinencia o se encontraba inmerso en un programa de sustitución de opioides.

El grupo de participantes estuvo formado por 41 hombres y 8 mujeres policonsumidores de drogas con una media de edad de 32.67 años $(S D=8.032)$ y de escolaridad de $10.46(S D=2.41)$. La duración de la abstinencia (para todas las drogas excepto para el tabaco) fue de 32.94 semanas ( $S D$ = 11.252). La Tabla 1 muestra los patrones de consumo de los participantes. Sólo se muestran los datos de aquellas drogas que habían sido consumidas por más del $15 \%$ de los participantes. De los 49 participantes, 46 habían consumido cocaína, 16 heroína, 45 alcohol, 23 MDMA y 37 cannabis.

\section{Herramientas}

Información del consumo de drogas. La información sobre el consumo de drogas se recogió utilizando el Inventario de investigación de la conducta adictiva (Verdejo-García, López-Torrecillas, Aguilar de Arcos, y Pérez-García, 2005). Este instrumento permite recoger información sobre la cantidad de cada droga consumida, la duración del consumo 
Tabla 1

Puntuaciones descriptivas de los patrones de cantidad y duración del consumo de drogas de los participantes

\begin{tabular}{lrccc}
\hline & \multicolumn{3}{c}{ Cantidad $^{\text {a }}$} & \\
\hline Sustancia & Media & D.T. & Máximo & Minimo \\
Cocaína & 48.69 & 44.83 & 180 & 0 \\
Heroína & 9.12 & 20.63 & 120 & 0 \\
Alcohol & 571.24 & 489.34 & 1800 & 0 \\
MDMA & 13.41 & 24.31 & 120 & 0 \\
Cannabis & 148.57 & 190.64 & 750 & 0 \\
\hline & & & Máximo & Minimo \\
\hline Sustancia & Media & Duración & 0 \\
Cocaína & 7.95 & 5.95 & 23 & 0 \\
Heroína & 1.76 & 3.69 & 17 & 0 \\
Alcohol & 10.85 & 7.67 & 27 & 0 \\
MDMA & 1.40 & 2.28 & 8 & 0 \\
Cannabis & 8.31 & 8.13 & 29 & 0 \\
\hline
\end{tabular}

Nota: a La cantidad de cada droga consumida es el resultado de multiplicar la cantidad media de cada una de las sustancias consumidas en cada episodio de consumo por frecuencia del consumo en un mes. b Duración del consumo en años.

o la duración de la abstinencia entre otras variables. Para evaluar estas variables se preguntó a cada uno de los participantes por la cantidad de droga consumida en un episodio de consumo regular (número de porros para cannabis, número de gramos de cocaína y heroína, y número de unidades de alcohol, considerando un combinado como dos unidades y un vaso de vino o cerveza como una), la frecuencia media del consumo (diaria, entre una y tres veces a la semana, una vez a la semana, entre una y tres veces al mes o una vez al mes), la duración del consumo en años y el número de semanas en situación de abstinencia (para todas las drogas excepto el tabaco). La cantidad de droga consumida se calculó multiplicando la cantidad media de cada una de las sustancias consumidas por la frecuencia del consumo al mes.

Adquisición de las imágenes. Las imágenes fueron adquiridas con un escáner ECAT/931 (Siemens CTI ECAT EXACT) situado en el servicio de Medicina Nuclear del hospital Virgen de las Nieves de Granada. La duración del escáner fue de 20 minutos. Esta sesión se llevó a cabo tras la administración de $200 \mathrm{MBq}$ de 18F-fluordesoxiglucosa (18-FDG) administrados tras comprobar que los niveles de glucemia se encontraban por debajo de $120 \mathrm{mg} / \mathrm{dl}$ (Claeys, Mertens, D'Asseler y Goethals, 2010). Los participantes fueron escaneados con los ojos abiertos y los oídos tapados en una habitación aislada acústicamente y con baja iluminación. Los datos fueron procesados usando una reconstrucción iteractiva OSEM con 10 repeticiones y 32 subconjuntos, y las imágenes fueron reorientadas en los planos transaxial, coronal y sagital.

\section{Análisis de las imágenes}

Pre-procesamiento de las imágenes. Las imágenes fueron convertidas de DICOM a NIFTI y normalizadas utilizando un procedimiento en dos pasos. En primer lugar, las imágenes fueron normalizadas utilizando la plantilla de PET del programa de análisis de imágenes Statistical Parametric Mapping - versión 5 (SPM5) (Wellcome Department of Cognitive Neurology, London, UK). A continuación, se creó una plantilla con las imágenes que habían sido normalizadas que actuó como plantilla de normalización para las imágenes (no normalizadas). De esta manera, las imágenes fueron normalizadas utilizando una plantilla que había sido creada utilizando las imágenes de nuestro grupo de participantes. En ambos casos las imágenes fueron normalizadas utilizando parámetros lineales y no lineales de normalización. Finalmente, las imágenes fueron suavizadas utilizando un filtro gaussiano de $8 \mathrm{~mm}^{3}$.

Análisis de regiones de interés. Tras llevar a cabo el pre-procesamiento de las imágenes, utilizamos la herramienta Marsbar (http://marsbar.sourceforge.net- Brett, Anton, Valabregue y Poline, 2002) para extraer los valores del consumo de la glucosa de 90 regiones de interés y AAL como atlas de referencia (Tzourio-Mazoyer et al., 2002). Para extraer los valores de consumo de glucosa utilizamos la opción "Extract ROI data (full options)" del menú "Data..." y a continuación seleccionamos las imágenes de todos nuestros pacientes. A continuación, los valores de la glucosa consumida en cada una de las regiones de interés fueron incluidos en un análisis de correlación bivariado utilizando el programa estadístico SPSS 15 y un nivel de significación de $\mathrm{p}<.05$. 


\section{Resultados}

Los resultados mostraron la presencia de correlaciones significativas entre la duración de la abstinencia y varias regiones de interés. Encontramos una correlación negativa entre la duración de la abstinencia y la amígdala (izquierda $r=-.291, p=.045$; derecha $r=-.355, \mathrm{p}=.013$ ) (Figura 1) y el hipocampo bilateralmente (izquierda $r=-.313, p=.031$; de- recha $r=-.307, p=.034$ ) (Figura 2) y una correlación positiva entre la duración de la abstinencia y la porción opercular del giro frontal inferior izquierdo $(r=.293, p=.043)$ (Figura 3$)$. No se encontraron asociaciones significativas entre la duración de la abstinencia y el resto de regiones de interés analizadas utilizando un nivel de significación de $\mathrm{p}<.05$.
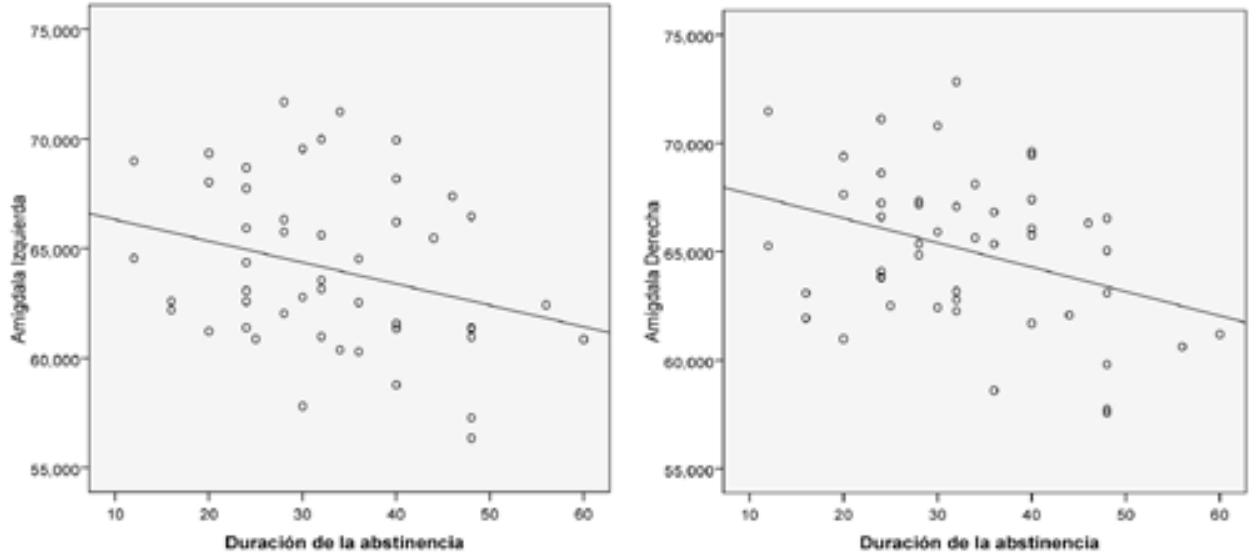

Figura 1. Correlación negativa entre el metabolismo cerebral de la amígdala y la duración de la abstinencia
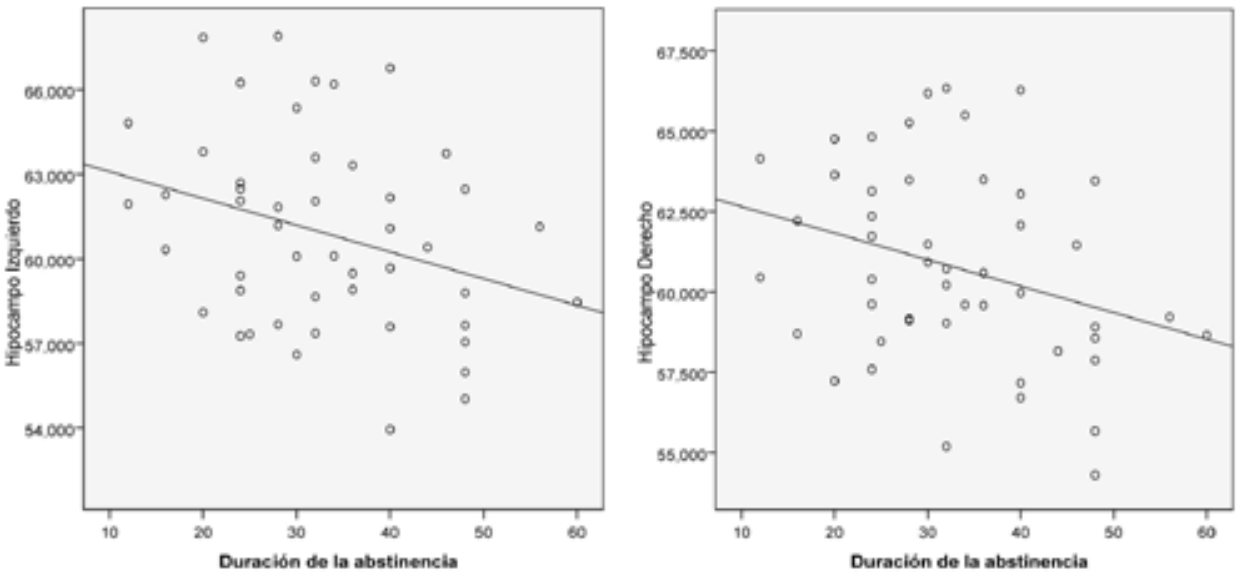

Figura 2. Correlación negativa entre el metabolismo cerebral del hipocampo y la duración de la abstinencia

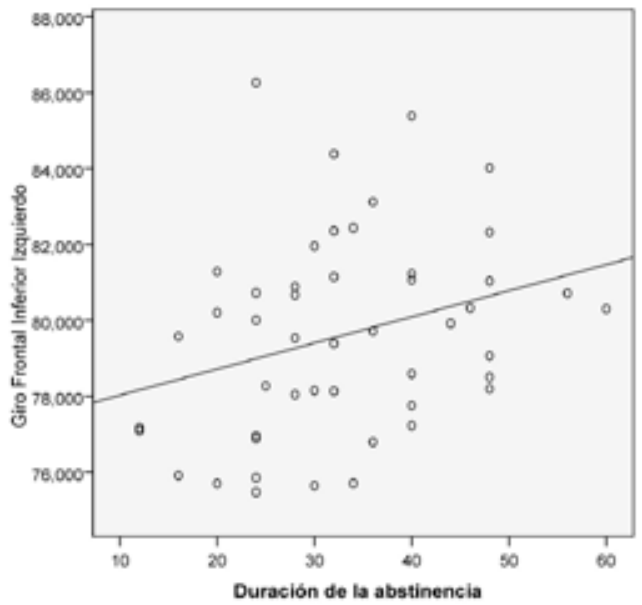

Figura 3. Correlación positiva entre el metabolismo cerebral de la región opercular del giro frontal interior y la duración de la abstinencia 


\section{Discusión}

El estudio de la asociación entre la duración de la abstinencia y el metabolismo de la glucosa en situación de abstinencia mostró la existencia de correlaciones significativas entre la duración de la abstinencia y el metabolismo de la glucosa de la amígdala y el hipocampo bilateralmente y la porción opercular del giro frontal inferior izquierdo. Las correlaciones fueron negativas en el primer caso y positivas en el segundo, sugiriendo una asociación diferencial entre estas estructuras y el tiempo de abstinencia.

La importancia de estos resultados radica no tanto en el cambio que haya podido producirse como consecuencia de la abstinencia en estas estructuras, pues estos cambios han sido reportados por aquellos estudios que han tratado de evaluar los efectos de la abstinencia tanto a nivel estructural como funcional, sino por la posible implicación diferencial de estas estructuras en el proceso adictivo.

La asociación negativa encontrada entre la duración de la abstinencia y la amígdala y el hipocampo podría ser el resultado de la hiperactivación de estas estructuras durante el consumo de las sustancias (Wise, 2009; Goldman et al., 2013; Grant et al., 1996) y su hipoactivación como resultado de la abstinencia. Una idea que coincide con un reciente estudio llevado a cabo por Bustamante y colaboradores en el que se encontró una asociación negativa entre la activación del estriado ventral producida por la presentación de estímulos reforzantes y la duración de la abstinencia de un grupo de consumidores de cocaína (Bustamante et al., en prensa). Es más, el hipometabolismo encontrado en la amígdala y el hipocampo podría indicar la alteración del sistema dopaminérgico y estar a la base de la disminución de la sensibilidad a reforzadores naturales mostrada por estos pacientes (Volkow, Fowler y Wang, 2003), así como los estados de ansiedad y estrés que caracterizan la abstinencia y que han sido asociados con el craving y las recaídas (Belujon y Grace, 2011; Dagher, Tannenbaum, Hayashi, Pruessner y McBride, 2009; García-Fuster, Flagel, Mahmood, Watson y Akil, 2012; Volkow, Fowler, Wang y Goldstein, 2002; Vorel, Liu, Hayes, Spector y Gardner, 2001; Wrase et al., 2008).

Por otra parte, la asociación positiva encontrada entre la duración de la abstinencia y el metabolismo de la glucosa de la porción opercular del giro frontal inferior indica que esta estructura pudo estar hipoactivada durante el consumo de drogas e hiperactivada como resultado de la abstinencia (Connolly et al., 2012; de Ruiter, Oosterlaan, Veltman, van den Brink y Goudriaan, 2012; Nestor, Ghahremani, Monterosso y London, 2011). Una hiperactivación o normalización del metabolismo cerebral en esta región que coincide con el papel de esta estructura en el control inhibitorio necesario para mantener la abstinencia (Connolly et al., 2012; Garavan y Weierstall, 2012; Volkow et al., 2010). En este sentido, la corteza frontal inferior ha sido consistentemente asociada con el control de la conducta (Aron, Fletcher, Bullmore, Sahakian y Robbins, 2003; Aron, Monsell, Sahakian y Rob- bins, 2004; Aron et al., 2007; Barrós-Loscertales et al., 2011; Garavan, Kaufman y Hester, 2008) y aunque la mayoría de los estudios de neuroimagen cerebral se han centrado en el papel de la corteza frontal inferior derecha, recientemente se ha demostrado que la parte izquierda también juega un papel fundamental en este proceso neuropsicológico (Connolly et al., 2012; Swick, Ashley y Turken, 2008). Es más, la parte izquierda del giro frontal inferior ha sido encontrada alterada en diversos consumidores de drogas (Moeller et al., 2010; Moreno-López et al., 2012c; Volkow et al., 2011) y ha sido asociada tanto con la impulsividad mostrada por sujetos sanos en tareas de descuento asociado a la demora o delay discount (Bjork, Momenan, y Hommer, 2009; Figner et al., 2010; McClure, Laibson, Loewenstein y Cohen, 2004) como con el craving de consumidores de cocaína y metanfetamina (Garavan et al., 2000; Tabibnia et al., 2011).

El hecho de que el metabolismo cerebral de estas estructuras cambie en función del tiempo de abstinencia tiene importantes implicaciones de cara a la rehabilitación del paciente drogodependiente, y es que, estas estructuras han sido asociadas con aspectos del proceso adictivo como la respuesta al estrés, la activación ante la exposición a estímulos condicionados con las drogas o la alteración del control inhibitorio que pueden llevar a la búsqueda de drogas y a la recaída, y que por lo tanto, deben ser tenidos en cuenta aún tras importantes periodos de abstinencia. La evidencia que indica la importancia del control cognitivo en la recaída del paciente drogodependiente proviene de los pocos estudios experimentales que han tratado de incrementar el control cognitivo en estos pacientes. En uno de estos estudios se encontró que el entrenamiento en autocontrol de un grupo de fumadores utilizando una tarea tan sencilla como evitar el consumo de dulces durante las dos semanas previas al abandono del tabaco traía consigo un incremento significativo de la abstinencia en este grupo al compararlo con la del grupo de fumadores que no recibió dicho entrenamiento (Muraven, 2010).

Por último, es importante destacar las fortalezas y limitaciones de este estudio. Entre sus fortalezas cabe destacar el tamaño de la muestra reclutada y el importante periodo de abstinencia del grupo de la misma. Respecto a las limitaciones, podría afirmarse que una limitación de este estudio es no contar con una muestra de controles no consumidores pero debido a que el objetivo de este estudio era conocer la asociación entre la duración de la abstinencia y el metabolismo de la glucosa, el contar con un grupo control no fue considerado necesario. Por otra parte, es importante destacar que factores como el policonsumo, el consumo de tabaco o la duración del tratamiento de cada uno de los participantes del estudio no fueron controlados y podrían estar influyendo en los resultados, así como el tipo de análisis realizado, pues al no incluir correcciones por múltiples comparaciones, puede habernos llevado a cometer error Tipo I. Finalmente, es importante destacar que este estudio 
no puede revelar si las alteraciones encontradas son el resultado del consumo de drogas o reflejan una característica previa del drogodependiente. Por lo que, estudios longitudinales que traten de dilucidar esta cuestión son altamente recomendables.

El resultado de este estudio apunta a la existencia de una implicación diferencial del hipocampo, la amígdala y la porción opercular del giro frontal inferior en el proceso adictivo indicado por las diferentes correlaciones encontradas entre la duración de la abstinencia y el metabolismo cerebral de la glucosa de dichas áreas. Este resultado tiene importantes implicaciones de cara al tratamiento porque esta información (la información acerca de la asociación de ciertas áreas cerebrales con la duración de la abstinencia) puede guiar al terapeuta en la selección de las estrategias o técnicas de tratamiento más adecuadas.

\section{Conflicto de intereses}

Los autores declaran no tener conflictos de intereses.

\section{Reconocimientos}

La investigación descrita en este trabajo ha sido financiada por un proyecto del Ministerio de Educación a través del Plan Nacional FPU (referencia AP2007-03583) y por el proyecto COPERNICO a través del Plan Nacional sobre Drogas (2009) para el último autor.

\section{Referencias}

Aron, A. R., Fletcher, P. C., Bullmore, E. T., Sahakian, B. J. y Robbins, T. W. (2003). Stop-signal inhibition disrupted by damage to right inferior frontal gyrus in humans. $\mathrm{Na}$ ture Neuroscience, 6, 115-116. doi: 10.1038/nn1003.

Aron, A. R., Monsell, S., Sahakian, B. J. y Robbins, T. W. (2004). A componential analysis of task-switching deficits associated with lesions of left and right frontal cortex. Brain, 127, 1561-1573. doi: 10.1093/brain/awh169.

Aron, A. R., Durston, S., Eagle, D. M., Logan, G. D., Stinear, C. M. y Stuphorn, V. (2007). Converging evidence for a fronto-basal-ganglia network for inhibitory control of action and cognition. The Journal of Neuroscience, 27, 11860-11864. doi: 10.1523/jneurosci.3644-07.2007.

Barrós-Loscertales, A., Bustamante, J. C., Ventura-Campos, N., Llopis, J. J., Parcet, M. A. y Avila, C. (2011). Lower activation in the right frontoparietal network during a counting Stroop task in a cocaine-dependent group. Psychiatry research, 194, 111-118. doi: 10.1016/j.pscychresns.2011.05.001.

Belujon, P. y Grace, A. A. (2011). Hippocampus, amygdala, and stress: interacting systems that affect susceptibility to addiction. Annals of the New York Academy of Sciences, 1216, 114-121. doi: 10.1111/j.1749-6632.2010.05896.x.
Bjork, J. M., Momenan, R. y Hommer, D. W. (2009). Delay discounting correlates with proportional lateral frontal cortex volumes. Biological Psychiatry, 65, 710-713. doi: 10.1016/j.biopsych.2008.11.023.

Brett, M., Anton, J-L., Valabregue, R. y Poline, J-B. (2002, junio). Region of interest analysis using an SPM toolbox. Comunicación presentada en la 8 a Conferencia Internacional de Human Brain Mapping, Japón.

Bustamante, J. C., Barrós-Loscertales, A., Costumero, V., Fuentes-Claramonte, P., Rosell-Negre, P., Ventura-Campos $\mathrm{N}, \ldots$ Avila, C. (en prensa). Abstinence duration modulates striatal functioning during monetary reward processing in cocaine patients. Addiction Biology. doi: 10.1111/adb.12041.

Camí, J. y Farré, M. (2003). Drug addiction. The New England journal of medicine, 349, 975-986. doi: 10.1056/ NEJMra023160.

Connolly, C. G., Foxe, J. J., Nierenberg, J., Shpaner, M. y Garavan, H. (2012). The neurobiology of cognitive control in successful cocaine abstinence. Drug and Alcohol Dependence, 121, 45-53. doi: 10.1016/j.drugalcdep.2011.08.007.

Claeys, J., Mertens, K., D’Asseler, Y. y Goethals, I. (2010). Normoglycemic plasma glucose levels affect F-18 FDG uptake in the brain. Annals of nuclear medicine, 24, 501505. doi: 10.1007/s12149-010-0359-9.

Dagher, A., Tannenbaum, B., Hayashi, T., Pruessner, J. C. y McBride, D. (2009). An acute psychosocial stress enhances the neural response to smoking cues. Brain research, 1293, 40-48. doi: 10.1016/j.brainres.2009.07.048.

de Ruiter, M. B., Oosterlaan, J., Veltman, D. J., van den Brink, W. y Goudriaan, A. E. (2012). Similar hyporesponsiveness of the dorsomedial prefrontal cortex in problem gamblers and heavy smokers during an inhibitory control task. Drug and Alcohol Dependence, 121, 81-89. doi: 10.1016/j.drugalcdep.2011.08.010.

Figner, B., Knoch, D., Johnson, E. J., Krosch, A. R., Lisanby, S. H., Fehr, E. y Weber, E. U. (2010). Lateral prefrontal cortex and self-control in intertemporal choice. Nature Neuroscience, 13, 538-539. doi: 10.1038/nn.2516.

First, M. B., Spitzer, R. L., Gibbon, M., Williams, J. B. W. (1997). Structured Clinical Interview for DSM-IV Axis I disorders (SCID I). New York: Biometric Research Department.

Garavan, H., Kaufman, J. N. y Hester, R. (2008). Acute effects of cocaine on the neurobiology of cognitive control. Philosophical Transactions of the Royal Society of London. Series B. Biological Science, 363, 3267-3276. doi: 10.1098/rstb.2008.0106.

Garavan, H., Pankiewicz, J., Bloom, A., Cho, J. K., Sperry, L., Ross, T. J.,... Stein, E. A. (2000). Cue-induced cocaine craving: neuroanatomical specificity for drug users and drug stimuli. The American Journal of Psychiatry, 157, 1789-1798. doi: 10.1176/appi.ajp.157.11.1789. 
Garavan, H. y Weierstall, K. (2012). The neurobiology of reward and cognitive control systems and their role in incentivizing health behavior. Preventive Medicine, 55, S17-23. doi: 10.1016/j.ypmed.2012.05.018.

García-Fuster, M. J., Flagel, S. B., Mahmood, S. T., Watson, S. J. y Akil, H. (2012). Cocaine withdrawal causes delayed dysregulation of stress genes in the hippocampus. PLoS One, 7, e42092. doi: 10.1371/journal.pone.0042092.

Goldman, M., Szucs-Reed, R. P., Jagannathan, K., Ehrman, R. N., Wang, Z., Li, Y.,... Franklin, T. R. (2013). Reward-related brain response and craving correlates of marijuana cue exposure: a preliminary study in treatment-seeking marijuana-dependent subjects. Journal of Addiction Medicine, 7, 8-16. doi: 10.1097/adm. 0b013e318273863a.

Goldstein, R. Z. y Volkow, N. D. (2011). Dysfunction of the prefrontal cortex in addiction: neuroimaging findings and clinical implications. Nature Reviews. Neuroscience 12, 652-669. doi: 10.1038/nrn3119.

Grant, S., London, E. D., Newlin, D. B., Villemagne, V. L., Liu, X., Contoreggi, C.,... Margolin, A. (1996). Activation of memory circuits during cue-elicited cocaine craving. Proceedings of the National Academy of Science of the United States of America, 93, 12040-12045. doi: 10.1073/ pnas.93.21.12040.

McClure, S. M., Laibson, D. I., Loewenstein, G. y Cohen, J. D. (2004). Separate neural systems value immediate and delayed monetary rewards. Science, 306, 503-507. doi: 10.1126/science. 110090 .

Moreno-López, L., Stamatakis, E. A., Fernández-Serrano, M. J., Gómez-Río, M., Rodríguez-Fernández, A., Pérez-García, M. y Verdejo-García A. (2012a). Neural correlates of hot and cold executive functions in polysubstance addiction: association between neuropsychological performance and resting brain metabolism as measured by positron emission tomography. Psychiatry Research, 203, 214-21. doi: 10.1016/j.pscychresns.2012.01.006.

Moreno-López, L., Stamatakis, E. A., Fernández-Serrano, M. J., Gómez-Río, M., Rodríguez-Fernández, A., Pérez-García, M. y Verdejo-García, A. (2012b). Neural correlates of the severity of cocaine, heroin, alcohol, MDMA and cannabis use in polysubstance abusers: a resting-PET brain metabolism study. PLoS One, 7, e39830. doi: 10.1371/journal.pone.0039830.

Moreno-López, L., Catena, A., Fernández-Serrano, M. J., Delgado-Rico, E., Stamatakis, E. A., Pérez-García, M. y Verdejo-García, A. (2012c). Trait impulsivity and prefrontal gray matter reductions in cocaine dependent individuals. Drug and Alcohol Dependence, 125, 208-214. doi: 10.1016/j.drugalcdep.2012.02.012.

Muraven, M. (2010). Practicing self-control lowers the risk of smoking lapse. Psychology of Addictive Behaviors, 24, 446-452. doi: 10.1037/a0018545.
Nestor, L. J., Ghahremani, D. G., Monterosso, J. y London, E. D. (2011). Prefrontal hypoactivation during cognitive control in early abstinent methamphetamine-dependent subjects. Psychiatry Research, 194, 287-295. doi: 10.1016/j.pscychresns.2011.04.010.

Swick, D., Ashley, V. y Turken, A. U. (2008). Left inferior frontal gyrus is critical for response inhibition. BMC neuroscience, 9, 102. doi: 10.1186/1471-2202-9-102.

Tabibnia, G., Monterosso, J. R., Baicy, K., Aron, A. R., Poldrack, R. A., Chakrapani, S.,... London, E. D. (2011). Different forms of self-control share a neurocognitive substrate. Journal of Neuroscience, 31, 4805-4810. doi: 10.1523/jneurosci.2859-10.2011.

Tapert, S. F., Schweinsburg, A. D., Drummond, S. P. A., Paulus, M. P., Brown, S. A., Yang, T. T. y Frank, L. R. (2007). Functional MRI of inhibitory processing in abstinent adolescent marijuana users. Psychopharmacology, 194, 173-183. doi: 10.1007/s00213-007-0823-y.

Tzourio-Mazoyer, N., Landeau, B., Papathanassiou, D., Crivello, F., Etard, O., Delcroix, N.,... Joliot, M. (2002). Automated anatomical labeling of activations in SPM using a macroscopic anatomical parcellation of the MNI MRI single-subject brain. NeuroImage, 15, 273-289. doi: 10.1006/nimg.2001.0978.

Verdejo-García, A. J., López-Torrecillas, F., Aguilar de Arcos, F. y Pérez-García, M. (2005). Differential effects of MDMA, cocaine, and cannabis use severity on distinctive components of the executive functions in polysubstance users: a multiple regression analysis. Addictive behaviors, 30, 89-101. doi: 10.1016/j.addbeh.2004.04.015.

Volkow, N. D., Fowler, J. S., Wang, G. J. y Goldstein, R. Z. (2002). Role of dopamine, the frontal cortex and memory circuits in drug addiction: insight from imaging studies. Neurobiology of Learning and Memory, 78, 610-624. doi: 10.1006/nlme.2002.4099.

Volkow, N. D., Fowler, J. S., Wang, G. J., Telang, F., Logan, J., Jayne, M.,... Swanson, J. M. (2010). Cognitive control of drug craving inhibits brain reward regions in cocaine abusers. Neuroimage, 49, 2536-2543. doi: 10.1016/j.neuroimage.2009.10.088.

Volkow, N. D., Fowler, J. S. y Wang, G. J. (2003). The addicted human brain: insights from imaging studies. The Journal of Clinical Investigation, 111, 1444-1451 doi: $10.1172 /$ jci200318533.

Volkow, N. D. y Lee, T. K. (2004). Drug addiction: the neurobiology of behavior gone awry. Nature reviews. Neuroscience, 5, 963-970. doi: 10.1038/nrn1539.

Volkow, N. D., Tomasi, D., Wang, G.-J., Fowler, J. S., Telang, F.,... Wong, C. (2011). Reduced metabolism in brain "control networks" following cocaine-cues exposure in female cocaine abusers. PloS one, 6, e16573. doi: 10.1371/journal.pone.0016573.

Vorel, S. R, Liu, X., Hayes, R. J., Spector, J. A. y Gardner, E. L. (2001). Relapse to cocaine-seeking after hippocam- 
pal theta burst stimulation. Science, 292, 1175-1178. doi: 10.1126 /science.1058043.

Wise, R. A. (2009). Roles for nigrostriatal-not just mesocorticolimbic-dopamine in reward and addiction. Trends in Neuroscience, 32, 517-524. doi: 10.1016/j. tins.2009.06.004.

Wrase, J., Makris, N., Braus, D. F., Mann, K., Smolka, M. N., Kennedy, D. N.,... Heinz, A. (2008). Amygdala vo- lume associated with alcohol abuse relapse and craving. The American Journal of Psychiatry, 165, 1179-1184. doi: 10.1176/appi.ajp.2008.07121877. 